\title{
Early Onset Non-Syndromic Obesity- Is Next Generation Sequencing the Next in Agenda?
}

\author{
Anurag Bajpai ${ }^{1}$ \\ Received: 24 December 2019 / Accepted: 24 December 2019 /Published online: 10 January 2020 \\ (C) Dr. K C Chaudhuri Foundation 2020
}

Childhood obesity is emerging as a major public health problem with doubling of prevalence over the last couple of decades [1]. While lifestyle factors are the main cause, genetic etiology remains an important consideration in early onset non-syndromic obesity. Clinical practice guidelines suggest genetic testing for children below the age of five years with non-syndromic severe obesity [2]. The diagnosis of these disorders had been challenging due to lack of clinical pointers and access to genetic testing.

In this issue of the journal, Khadilkar et al., report their experience of Next generation genetic testing for two important obesity related genes, Leptin and MC4 receptor in 46 children with early onset non-syndromic obesity [3]. They identified pathological mutations in 4 (8.5\%; Leptin deficiency in 3 and MC4 Receptor defects in 1) and emphasized the importance of genetic studies in these children for prognosis, treatment and genetic counseling.

The study reaffirms the importance of genetic testing in determining etiology of early onset non-syndromic obesity. Universal screening of children below the age of five years with severe non-syndromic obesity for genetic etiology however poses a substantial resource burden. Moreover, it is expected to unearth etiology in only $8.5 \%$ for which effective treatment is either not available or accessible. Inclusion of greater number of target genes would have increased the diagnostic yield of the study, however, at a substantially greater cost. This suggests the need for selective evaluation of subjects with high pretest probability of ge-

Anurag Bajpai

dr_anuragbajpai@yahoo.com

1 Pediatric \& Adolescent Endocrinology, Regency Center for Diabetes, Endocrinology \& Research, Kanpur, Uttar Pradesh, India netic obesity. Findings of this study suggest that age at presentation is an important predictor of genetic etiology in these children. All subjects with genetic obesity in this study were younger than $2.5 \mathrm{y}$. This along with 9.5 times odds of children below the age of two years having genetic etiology than older subjects, indicate the need for lowering age threshold for genetic evaluation.

In conclusion, the study reiterates the importance of genetic testing in children with non-syndromic early onset obesity. There is a need for larger studies with inclusion of greater number of obesity genes to determine the contribution of genetic causes in non-syndromic early onset obesity in Indian children and devising protocols for targeted evaluation.

\section{Compliance with Ethical Standards}

Conflict of Interest None.

\section{References}

1. Eshwar TKM, Chudasama RK, Eshwar ST, Thakrar D. Prevalence of obesity and overweight and their comparison by three growth standards among affluent school students aged 8-18 years in Rajkot. Indian J Public Health. 2017;61:51-4.

2. Styne DM, Arslanian SA, Connor EL, et al. Pediatric obesity-assessment, treatment, and prevention: an Endocrine Society Clinical Practice Guideline. J Clin Endocrinol Metab. 2017;102:709-57.

3. Khadilkar V, Gogate N, Gangodkar P, et al. A targeted next generation sequencing panel for nonsyndromic early onset severe obesity and identification of novel likely pathogenic variants in the MC4R and LEP genes. Indian J Pediatr. 2019. https://doi.org/10.1007/ s12098-019-03129-6.

Publisher's Note Springer Nature remains neutral with regard to jurisdictional claims in published maps and institutional affiliations. 